\title{
NEUMANN AND ROBIN TYPE BOUNDARY CONDITIONS IN FRACTIONAL ORLICZ-SOBOLEV SPACES
}

\author{
SABri BAHrouni ${ }^{1}$ AND ARIEL M. SAlorT ${ }^{2, *}$
}

\begin{abstract}
In the first part of this article we deal with the existence of at least three non-trivial weak solutions of a nonlocal problem with nonstandard growth involving a nonlocal Robin type boundary condition. The second part of the article is devoted to study eigenvalues and minimizers of several nonlocal problems for the fractional $g$-Laplacian $\left(-\Delta_{g}\right)^{s}$ with different boundary conditions, namely, Dirichlet, Neumann and Robin.
\end{abstract}

Mathematics Subject Classification. 46E30, 35R11, 45G05.

Received March 30, 2020. Accepted September 29, 2020.

\section{INTRODUCTION}

In the recent years has been an increasing interest in studying non-local problems with $p$-structure due to its accurate description of models involving anomalous diffusion. In several branches of science have been observed some phenomena having a non-local nature, which, nonetheless, do not obey a power-like growth law. See for instance $[2,3,6,20,23]$ and references therein.

The suitable operator to describe these kind of phenomena is the fractional $g$-Laplacian introduced in [20] and defined as

$$
\left(-\Delta_{g}\right)^{s} u:=\text { p.v. } \int_{\mathbb{R}^{n}} g\left(\left|D_{s} u\right|\right) \frac{D_{s} u}{\left|D_{s} u\right|} \frac{\mathrm{d} y}{|x-y|^{n+s}},
$$

and defined in the principal value sense; here $G$ is a Young function such that $g=G^{\prime}$ and $s \in(0,1)$ is a fractional parameter. The quantity $D_{s} u:=\frac{u(x)-u(y)}{|x-y|^{s}}$ is the $s-H \ddot{o l d e r}$ quotient.

Problems involving this operator have recently attracted some attention. We refer the readers to [3-5, $7-$ $10,16,20,21,35]$. Observe that when $G(t)=t^{p} / p, p>1$, (1.1) becomes the well-known fractional p-Laplacian operator. See also [13] for a non-singular version.

Given an open bounded domain $\Omega \subset \mathbb{R}^{n}$ with smooth boundary ( $\partial \Omega \in C^{0,1}$ is enough) the first aim of the present article is to study existence of nontrivial solutions of the following equation involving the nonlinearities

Keywords and phrases: Fractional Orlicz-Sobolev spaces, Neumann and Robin problem, three solutions, eigenvalue problems.

1 Department of Mathematics, Faculty of Sciences, University of Monastir, 5019 Monastir, Tunisia.

2 Departamento de Matemática, FCEyN - Universidad de Buenos Aires and IMAS - ICET Ciudad Universitaria, Pabellón I

(1428) Av. Cantilo s/n. Buenos Aires, Argentina.

* Corresponding author: asalort@dm.uba.ar 
$f$ and $h$ with homogeneous Robin boundary condition on $\mathbb{R}^{n} \backslash \Omega$

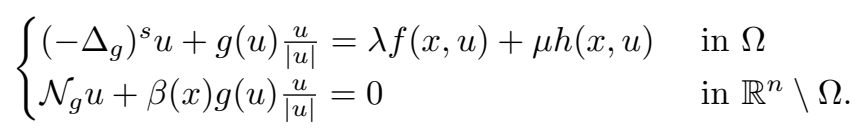

Here, we introduce a non-local normal derivative in this settings as

$$
\mathcal{N}_{g} u(x):=\int_{\Omega} g\left(\left|D_{s} u\right|\right) \frac{D_{s} u}{\left|D_{s} u\right|} \frac{\mathrm{d} y}{|x-y|^{n+s}}, \quad x \in \mathbb{R}^{n} \backslash \bar{\Omega}
$$

which can be seen as the natural generalization of the non-local derivative introduced in [17].

Nonlocal equations for the fractional $p$-Laplacian with boundary conditions involving non-local normal derivatives have been recently developed in the literature; see for instance $[1,14,15,17,18,31,37,38]$.

Regarding existence of solutions to problem (1.2) in the particular case of the fractional $p$-Laplacian, there has been some recent develops. In [31], under suitable conditions on the nonlinearities, the authors obtain existence of at most one positive solution by following the celebrated paper of Brezis-Oswald. The authors in [30], for the same problem but with $\beta \equiv 0$, and under suitable conditions on $f$, by using variational methods obtain existence of two positive solutions. It worths to be mention that the local counterpart of (1.2) for Orlicz functions in the Dirichlet case was studied in $[12,24,32]$. For some existence results in the nonlocal Orlicz case with Dirichlet boundary conditions see [5]. For problems with critical Trudinger-Moser nonlinearities see [28]. Finally, for an introduction to the theory of variational methods for nonlocal fractional problems we recommend [29].

Our first main scope is to provide conditions on the Young function $G$, on the nonlinearities $f$ and $h$, and over $\lambda, \mu$ and $\beta$ to ensure existence of at least three nontrivial (weak) solutions of (1.2). Our arguments are based in the celebrated result [34] by B. Ricceri together with an integration by parts formula related to the operator $\left(-\Delta_{g}\right)^{s}$.

The Young function $G=\int_{0}^{t} g(t) \mathrm{d} t$ is assumed to satisfy the following growing condition

$$
1<p^{-} \leq \frac{t g(t)}{G(t)} \leq p^{+}<\infty \quad \forall t>0
$$

for fixed constants $p^{ \pm}$. Moreover, the following structural condition is assumed

$$
t \mapsto G(\sqrt{t}), t \in[0, \infty[\text { is convex. }
$$

To ensure compactness we restrict ourselves to the sub-critical case of the fractional Orlicz-Sobolev embeddings:

$$
\int_{0}^{1} \frac{G^{-1}(\tau)}{\tau^{\frac{n+s}{n}}} \mathrm{~d} \tau<\infty \quad \text { and } \quad \int_{1}^{+\infty} \frac{G^{-1}(\tau)}{\tau^{\frac{n+s}{n}}} \mathrm{~d} \tau=\infty
$$

Here, $\lambda$ and $\mu$ are two positive real parameters in a suitable range and $\beta \in L^{\infty}\left(\mathbb{R}^{n} \backslash \Omega\right)$ is strictly positive. The nonlinearities $f, h: \Omega \times \mathbb{R} \rightarrow \mathbb{R}$ will be suitable Carathéodory functions assumed to belong to the class $\mathcal{A}$ defined as follows: $f \in \mathcal{A}$ if it fulfills the growth condition

$$
|f(x, t)| \leq w(x)(1+m(|t|)) \text { for a.e } x \in \Omega \text { and for all } t \in \mathbb{R},
$$

where $w$ is a positive function such that $w \in L^{\infty}(\Omega)$ and $m=M^{\prime}$, being $M$ a Young function decreasing essentially more rapidly than the critical Sobolev function $G_{*}$, i.e., $M \prec \prec G_{*}$, being $G_{*}$ the critical function in the fractional Orlicz-Sobolev embedding (see Sect. 2.2 for details). We remark that $\left(f_{1}\right)$ is fulfilled, for instance, if $|f(x, t)| \leq w(x)(1+|u|)^{q-1}$ for some $q \in\left(1, p_{*}^{-}\right)$, being $p_{*}^{-}:=\frac{n p^{-}}{n-p^{-}}$. 
From now on, we denote

$$
F(x, t)=\int_{0}^{t} f(x, s) \mathrm{d} s, \quad H(x, t)=\int_{0}^{t} h(x, s) \mathrm{d} s, \quad \mathcal{F}(u)=\int_{\Omega} F(x, u) \mathrm{d} x,
$$

and we anticipate that the natural space to look for (weak) solutions of (1.2) is given by (see Sect. 3 for details and motivations)

$$
\mathcal{X}=\left\{u \text { measurable }: \iint_{\mathbb{R}^{2 n} \backslash\left(\Omega^{c}\right)^{2}} G\left(\left|D_{s} u(x, y)\right|\right) \mathrm{d} \mu+\int_{\Omega} G(|u|) \mathrm{d} x+\int_{\mathbb{R}^{n} \backslash \Omega} \beta G(|u|) \mathrm{d} x<\infty\right\},
$$

where we have denoted $\mathrm{d} \mu:=\frac{\mathrm{d} x \mathrm{~d} y}{|x-y|^{n}}$.

With these preliminaries, our first result reads as follows.

Theorem 1.1. Let $G$ be a Young function satisfying the structural hypotheses $\left(G_{1}\right),\left(G_{2}\right)$ and $\left(G_{3}\right)$, let $\beta \in$ $L^{\infty}\left(\mathbb{R}^{n} \backslash \Omega\right)$ and let $f, h \in \mathcal{A}$ be such that

$$
\begin{gathered}
\max \left\{\limsup _{|u| \rightarrow 0} \frac{\sup _{x \in \Omega} F(x, u)}{G(u)}, \limsup _{|u| \rightarrow+\infty} \frac{\sup _{x \in \Omega} F(x, u)}{G(u)}\right\} \leq 0, \\
\sup _{u \in \mathcal{X}} \int_{\Omega} F(x, u) \mathrm{d} x>0 .
\end{gathered}
$$

Then, if we set

$$
\delta=\inf \left\{\frac{\mathcal{J}(u)}{\mathcal{F}(u)}: u \in \mathcal{X}, \mathcal{F}(u)>0\right\}
$$

where

$$
\mathcal{J}(u):=\iint_{\mathbb{R}^{2 n} \backslash\left(\Omega^{c}\right)^{2}} G\left(\left|D_{s} u\right|\right) \mathrm{d} \mu+\int_{\Omega} G(|u|) \mathrm{d} x+\int_{\mathbb{R}^{n} \backslash \Omega} \beta G(|u|) \mathrm{d} x,
$$

for each compact interval $[a, b] \subset(\delta, \infty)$ there exists $\nu>0$ with the following property: for every $\lambda \in[a, b]$ and $h$, there exists $\gamma>0$ such that, for each $\mu \in[0, \gamma]$, problem (1.2) has at least three weak solutions whose norms in $\mathcal{X}$ are less than $\nu$.

We also prove the following result characterizing the geometry involved in the class of admissible nonlinearities.

Theorem 1.2. Let $G$ be a Young function satisfying $\left(G_{1}\right),\left(G_{2}\right)$ and $\left(G_{3}\right)$, let $\beta \in L^{\infty}\left(\mathbb{R}^{n} \backslash \Omega\right)$ and let $f, h \in \mathcal{A}$ such that

(i) there exists a Young function $B(t)=\int_{0}^{t} b(\tau) \mathrm{d} \tau$ such that $\frac{t b(t)}{B(t)} \leq b^{+}<p^{-}$and $B \prec \prec G$, and a constant $c_{1}>0$ for which

$$
F(x, t) \leq c_{1}(1+B(t)) \quad \text { for all }(x, t) \in \Omega \times \mathbb{R}
$$


(ii) there exist a constant $c_{2}>0, \tau_{1}>0$ and a Young function $D(t)=\int_{0}^{t} \mathrm{~d}(\tau) \mathrm{d} \tau$ such that $p^{+}<\mathrm{d}^{-} \leq \frac{t \mathrm{~d}(t)}{D(t)}$ and $G \prec \prec D$ for which

$$
F(x, t) \leq c_{2} D(t) \quad \text { for all }(x, t) \in \Omega \times\left[-\tau_{1}, \tau_{1}\right]
$$

(iii) there exists $\tau_{2} \in \mathbb{R} \backslash\{0\}$ such that

$$
F\left(x, \tau_{2}\right)>0 \quad \text { and } F(x, t) \geq 0 \quad \text { for all }(x, t) \in \Omega \times\left[0, \tau_{2}\right] .
$$

Then there exists $\delta>0$ such that for every compact interval $[a, b] \subset(\delta, \infty)$ there exists a real number $\nu$ such that, for every $\lambda \in[a, b]$ and every continuous function $h$ there exists $\gamma>0$ such that, for each $\mu \in[0, \gamma]$, then problem (1.2) has at least three weak solutions whose norms in $\mathcal{X}$ are less than $\nu$.

We remark that the class of admissible nonlinearities in Theorem 1.1 includes perturbations of powers and concave-convex type combinations, among other. See Section 4 for further examples.

Very close to (1.2), as a second aim, we will study eigenvalues and minimizers of several nonlocal problems with non-standard growth involving different boundary conditions. For the case of powers, that is, for fractional $p$-Laplacian type operators, the Dirichlet case was studied for instance in $[27,36]$, for the Neumann case see for instance [15, 30], the Robin case was dealt in [19]. For general Orlicz functions and Dirichlet boundary conditions we refer to [35].

To be more precise, we consider the following Dirichlet eigenvalue problem

$$
\begin{cases}\left(-\Delta_{g}\right)^{s} u+g(|u|) \frac{u}{|u|}=\lambda g(|u|) \frac{u}{|u|} & \text { in } \Omega \\ u=0 & \text { in } \mathbb{R}^{n} \backslash \Omega,\end{cases}
$$

the following Neumann problem in terms of the nonlocal normal derivative $\mathcal{N}_{g}$

$$
\begin{cases}\left(-\Delta_{g}\right)^{s} u+g(|u|) \frac{u}{|u|}=\lambda g(|u|) \frac{u}{|u|} & \text { in } \Omega \\ \mathcal{N}_{g} u=0 & \text { in } \mathbb{R}^{n} \backslash \Omega,\end{cases}
$$

the following problem, which, from a probabilistic point of view can be seen also as a Neumann eigenvalue problem (see [15])

$$
\left\{\begin{array}{l}
\left(-\Delta_{g}\right)_{\Omega}^{s} u+g(|u|) \frac{u}{|u|}=\lambda g(|u|) \frac{u}{|u|} \quad \text { in } \Omega \\
u \in W_{\text {reg }}^{s, G},
\end{array}\right.
$$

and finally, the following Robin eigenvalue problem

$$
\begin{cases}\left(-\Delta_{g}\right)^{s} u+g(|u|) \frac{u}{|u|}=\lambda g(|u|) \frac{u}{|u|} & \text { in } \Omega \\ \mathcal{N}_{g} u+\beta g(|u|) \frac{u}{|u|}=0 & \text { in } \mathbb{R}^{n} \backslash \Omega .\end{cases}
$$

Here, for $0<s<1$ we have denoted the regional fractional $g$-Laplacian as

$$
\left(-\Delta_{g}\right)_{\Omega}^{s} u:=2 \text { p.v. } \int_{\Omega \times \Omega} g\left(\left|D_{s} u\right|\right) \frac{D_{s} u}{\left|D_{s} u\right|} \frac{\mathrm{d} y}{|x-y|^{n+s}},
$$


which is naturally defined in the space

$$
W_{\text {reg }}^{s, G}(\Omega):=\left\{u: \int_{\Omega} G(|u|) \mathrm{d} x+\iint_{\Omega \times \Omega} G\left(D_{s} u\right) \mathrm{d} \mu<\infty\right\} .
$$

A substantial difference which contrasts with the case of powers is that, in general, eigenvalues of (1.4), (1.5), (1.6) and (1.7) are not variational, i.e., they cannot be obtained by minimizing some Rayleigh quotient on a suitable space. For this reason, it is very interesting to study also the natural variational minimization problem related to Dirichlet, Neumann, regional Neumann and Robin boundary conditions. In order to not extend considerably the length of this introduction, we anticipate that the corresponding minimizers exist, are well defined (see Prop. 5.4) and are denoted as $\Lambda_{D}, \Lambda_{N}, \Lambda_{\tilde{N}}$ and $\Lambda_{R}$, respectively, but we will not define them here (see Eqs. (5.5), (5.6), (5.7) and (5.8) for the precise definition).

In spite of the fact that eigenvalues and minimizers are different quantities in general, in light of Proposition 5.8 they are comparable, with equality in the case of powers (i.e., when $G(t)=t^{p} / p, p>1$ ). Regarding the relation among the different minimizers, in Proposition 5.6 we prove that they are ordered as

$$
\Lambda_{\tilde{N}} \leq \Lambda_{N} \leq \Lambda_{R} \leq \Lambda_{D}
$$

In view of the aforementioned Proposition 5.8, eigenvalues are consequently ordered as

$$
\lambda_{\tilde{N}} \leq c^{2} \lambda_{N} \leq c^{4} \lambda_{R} \leq c^{6} \lambda_{D}
$$

where $c=p^{+} / p^{-}$.

In Theorem 5.5 we prove that a function reaching the minimization problem for $\Lambda \in\left\{\Lambda_{\tilde{N}}, \Lambda_{N}, \Lambda_{R}, \Lambda_{D}\right\}$ is an eigenfunction for $\lambda \in\left\{\lambda_{\tilde{N}}, \lambda_{N}, \lambda_{R}, \lambda_{D}\right\}$, respectively. A considerable difference with the case of powers is that, due to the non-homogeneous nature of the problems, both eigenvalues and minimizers strongly depend on the energy level: for each $\mu>0$, if the eigenfunction/minimizing function is normalized such that $\int_{\Omega} G(|u|)=\mu$, then $\Lambda$ and $\lambda$ depend on $\mu$. Nevertheless, in Proposition 5.7 we prove that $\Lambda$ and $\lambda$ are uniformly bounded by below independently of $\mu$.

Before concluding this introduction, we mention some interesting issues we not deal and let as open questions: to establish positivity of eigenfunctions, to obtain its boundedness, and to study its interior/up to the boundary regularity.

This paper is organized as follows. In Section 2 we introduce some preliminary results and definitions, as well as a proof of an integration by parts formula related to the operator $(-\Delta)_{g}^{s}$. Section 3 deals with the proof of our existence results. Some examples of nonlinearities which illustrate Theorems 1.1 and 1.2 are given in Section 4. Finally, Section 5 is devoted to study the eigenvalue problems (1.4), (1.5), (1.6) and (1.7).

\section{PreliminaRies}

In this section we introduce the classes of Young function and fractional Orlicz-Sobolev functions, the suitable class where the fractional $g$-Laplacian is well defined.

\subsection{Young functions}

An application $G: \mathbb{R}_{+} \rightarrow \mathbb{R}_{+}$is said to be a Young function if it admits the integral formulation $G(t)=$ $\int_{0}^{t} g(\tau) \mathrm{d} \tau$, where the right continuous function $g$ defined on $[0, \infty)$ has the following properties:

$$
\begin{aligned}
& g(0)=0, \quad g(t)>0 \text { for } t>0, \\
& g \text { is nondecreasing on }(0, \infty), \\
& \lim _{t \rightarrow \infty} g(t)=\infty
\end{aligned}
$$


From these properties it is easy to see that a Young function $G$ is continuous, nonnegative, strictly increasing and convex on $[0, \infty)$.

We will assume from now on that the Young functions satisfy the growth behavior given in $\left(G_{1}\right)$. Roughly speaking, this condition indicates that $G$ remains between two power functions.

The following properties on Young functions are well-known. See for instance [25] for a proof.

Lemma 2.1. Let $G$ be a Young function satisfying $\left(G_{1}\right)$ and $a, b \geq 0$. Then

$$
\begin{aligned}
& \min \left\{a^{p^{-}}, a^{p^{+}}\right\} G(b) \leq G(a b) \leq \max \left\{a^{p^{-}}, a^{p^{+}}\right\} G(b), \\
& G(a+b) \leq \mathbf{C}(G(a)+G(b)) \quad \text { with } \mathbf{C}:=2^{p^{+}} \\
& G \text { is Lipschitz continuous. }
\end{aligned}
$$

Condition $\left(L_{2}\right)$ is known as the $\Delta_{2}$ condition or doubling condition and, as it is showed in ([25], Thm. 3.4.4), it is equivalent to the right hand side inequality in $\left(G_{1}\right)$.

The complementary Young function $\tilde{G}$ of a Young function $G$ is defined as

$$
\tilde{G}(t):=\sup \{t w-G(w): w>0\} .
$$

From this definition the following Young-type inequality holds

$$
a b \leq G(a)+\tilde{G}(b) \quad \text { for all } a, b \geq 0,
$$

and the following Hölder's type inequality

$$
\int_{\Omega}|u v| \mathrm{d} x \leq\|u\|_{G}\|v\|_{\tilde{G}}
$$

for all $u \in L^{G}(\Omega)$ and $v \in L^{\tilde{G}}(\Omega)$. Moreover, it is not hard to see that $\tilde{G}$ can be written in terms of the inverse of $g$ as

$$
\tilde{G}(t)=\int_{0}^{t} g^{-1}(\tau) \mathrm{d} \tau
$$

see ([33], Thm. 2.6.8).

Since $\varphi^{-1}$ is increasing, from $(2.1)$ and $\left(G_{1}\right)$ it is immediate the following relation.

Lemma 2.2. Let $G$ be an Young function satisfying $\left(G_{1}\right)$ such that $g=G^{\prime}$ and denote by $\tilde{G}$ its complementary function. Then

$$
\tilde{G}(g(t)) \leq\left(p^{+}+1\right) G(t)
$$

holds for any $t \geq 0$.

The following convexity property will be useful.

Lemma 2.3. ([26], Lem. 2.1) Let $G$ be a Young function satisfying $\left(G_{1}\right)$ and $\left(G_{2}\right)$. Then for every a, $b \in \mathbb{R}$,

$$
\frac{G(|a|)+G(|b|)}{2} \geq G\left(\left|\frac{a+b}{2}\right|\right)+G\left(\left|\frac{a-b}{2}\right|\right) .
$$




\subsection{Fractional Orlicz-Sobolev spaces}

Given a Young function $G$, a parameter $s \in(0,1)$ and an open and bounded set $\Omega \subseteq \mathbb{R}^{n}$ we consider the spaces

$$
\begin{aligned}
& L^{G}(\Omega):=\left\{u: \Omega \rightarrow \mathbb{R} \text { measurable }: \Phi_{G, \Omega}(u)<\infty\right\} \\
& W^{s, G}(\Omega):=\left\{u \in L^{G}(\Omega): \Phi_{s, G, \mathbb{R}^{n}}(u)<\infty\right\} \\
& W_{\text {reg }}^{s, G}(\Omega):=\left\{u \in L^{G}(\Omega): \Phi_{s, G, \Omega}(u)<\infty\right\}
\end{aligned}
$$

where the modulars $\Phi_{G, \Omega}$ and $\Phi_{s, G}$ are defined as

$$
\begin{aligned}
& \Phi_{G, \Omega}(u):=\int_{\Omega} G(|u(x)|) \mathrm{d} x \\
& \Phi_{s, G, \mathbb{R}^{n}}(u):=\iint_{\mathbb{R}^{n} \times \mathbb{R}^{n}} G\left(\left|D_{s} u(x, y)\right|\right) \mathrm{d} \mu, \\
& \Phi_{s, G, \Omega}(u):=\iint_{\Omega \times \Omega} G\left(\left|D_{s} u(x, y)\right|\right) \mathrm{d} \mu,
\end{aligned}
$$

and the $s$-Hölder quotient is defined as

$$
D_{s} u(x, y):=\frac{u(x)-u(y)}{|x-y|^{s}}
$$

with $\mathrm{d} \mu(x, y):=\frac{\mathrm{d} x \mathrm{~d} y}{|x-y|^{n}}$. These spaces are endowed with the so-called Luxemburg norms

$$
\begin{aligned}
& \|u\|_{L^{G}(\Omega)}:=\inf \left\{\lambda>0: \Phi_{G, \Omega}\left(\frac{u}{\lambda}\right) \leq 1\right\}, \\
& \|u\|_{W^{s, G}(\Omega)}:=\|u\|_{L^{G}(\Omega)}+[u]_{W^{s, G}\left(\mathbb{R}^{n}\right)}, \\
& \|u\|_{W_{r e g}^{s, G}(\Omega)}:=\|u\|_{L^{G}(\Omega)}+[u]_{W_{\text {reg }}^{s, G}(\Omega)},
\end{aligned}
$$

where the $(s, G)$-Gagliardo semi-norms are defined as

$$
\begin{aligned}
& {[u]_{W^{s, G}\left(\mathbb{R}^{n}\right)}:=\inf \left\{\lambda>0: \Phi_{s, G, \mathbb{R}^{n}}\left(\frac{u}{\lambda}\right) \leq 1\right\},} \\
& {[u]_{W_{\text {reg }}^{s, G}(\Omega)}:=\inf \left\{\lambda>0: \Phi_{s, G, \Omega}\left(\frac{u}{\lambda}\right) \leq 1\right\} .}
\end{aligned}
$$

The space $W^{s, G}(\Omega)$ is a reflexive Banach space. Moreover $C_{c}^{\infty}$ is dense in $W^{s, G}\left(\mathbb{R}^{n}\right)$. See ([20], Prop. 2.11) and ([16], Prop. 2.9) for details.

We also consider the following space

$$
W_{0}^{s, G}(\Omega):=\left\{u \in W^{s, G}\left(\mathbb{R}^{n}\right): u=0 \text { a.e. in } \mathbb{R}^{n} \backslash \Omega\right\} .
$$

Observe that $W_{0}^{s, G}(\Omega) \subset W^{s, G}\left(\mathbb{R}^{n}\right) \subset L^{G}\left(\mathbb{R}^{n}\right)$.

In order to state some embedding results for fractional Orlicz-Sobolev spaces we introduce the following notation.

Given two Young functions $A$ and $B$, we say that $B$ is essentially stronger than $A$ or equivalently that $A$ decreases essentially more rapidly than $B$, and denoted by $A \prec \prec B$, if for each $a>0$ there exists $x_{a} \geq 0$ such that $A(x) \leq B(a x)$ for $x \geq x_{a}$. 
When the Young function $G$ fulfills condition $\left(G_{3}\right)$, the critical function for the fractional Orlicz-Sobolev embedding is given by

$$
G_{*}^{-1}(t)=\int_{0}^{t} \frac{G^{-1}(\tau)}{\tau^{\frac{n+s}{n}}} \mathrm{~d} \tau
$$

The following result can be found in [8]. See also [3] for further generalizations.

Theorem 2.4. Let $G$ be a Young function satisfying $\left(G_{3}\right)$ and $s \in(0,1)$. Let $\Omega \subset \mathbb{R}^{n}$ be a $C^{0,1}$ bounded open subset. Then

(i) the embedding $W_{\text {reg }}^{s, G}(\Omega) \hookrightarrow L^{G_{*}}(\Omega)$ is continuous;

(ii) for any Young function $B$ such that $B \prec \prec G_{*}$, the embedding $W_{\text {reg }}^{s, G}(\Omega) \hookrightarrow L^{B}(\Omega)$ is compact.

From (2.1) it follows the following relation between modulars and norms. See ([7], Lem. 3.1) or ([22], Lem. 2.1).

Lemma 2.5. Let $G$ be a Young function satisfying $\left(G_{1}\right)$ and let $\xi^{-}(t)=\min \left\{t^{p^{-}}, t^{p^{+}}\right\}, \xi^{+}(t)=\max \left\{t^{p^{-}}, t^{p^{+}}\right\}$, for all $t \geq 0$. Then, given $\Omega \subset \mathbb{R}^{n}$,

(i) $\xi^{-}\left(\|u\|_{G}\right) \leq \Phi_{G, \Omega}(u) \leq \xi^{+}\left(\|u\|_{G}\right)$ for $u \in L^{G}(\Omega)$,

(ii) $\xi^{-}\left([u]_{s, G}\right) \leq \Phi_{s, G, \Omega}(u) \leq \xi^{+}\left([u]_{s, G}\right)$ for $u \in W^{s, G}(\Omega)$.

\subsection{The fractional $g$-Laplacian operator}

Let $G$ be a Young function such that $G^{\prime}=g$ and $s \in(0,1)$. As anticipated, the fractional $g$-Laplacian operator is defined as

$$
\left(-\Delta_{g}\right)^{s} u:=2 \text { p.v. } \int_{\mathbb{R}^{n}} g\left(\left|D_{s} u\right|\right) \frac{D_{s} u}{\left|D_{s} u\right|} \frac{\mathrm{d} y}{|x-y|^{n+s}},
$$

where p.v. stands for in principal value. This operator is well defined between $W^{s, G}\left(\mathbb{R}^{n}\right)$ and its dual space $W^{-s, G^{*}}\left(\mathbb{R}^{n}\right)$. In fact, in ([20], Thm. 6.12) the following representation formula is provided

$$
\left\langle\left(-\Delta_{g}\right)^{s} u, v\right\rangle=\iint_{\mathbb{R}^{n} \times \mathbb{R}^{n}} g\left(\left|D_{s} u\right|\right) \frac{D_{s} u}{\left|D_{s} u\right|} D_{s} v \mathrm{~d} \mu,
$$

for any $v \in W^{s, G}\left(\mathbb{R}^{n}\right)$.

On the other hand, the censored or regional fractional $g$-Laplacian is well defined between $W_{r e g}^{s, G}(\Omega)$ and its dual space and it is defined as

$$
\left(-\Delta_{g}\right)_{\Omega}^{s} u:=2 \text { p.v. } \int_{\Omega} g\left(\left|D_{s} u\right|\right) \frac{D_{s} u}{\left|D_{s} u\right|} \frac{\mathrm{d} y}{|x-y|^{n+s}},
$$

which acts as

$$
\left\langle\left(-\Delta_{g}\right)_{\Omega}^{s} u, v\right\rangle=\iint_{\Omega \times \Omega} g\left(\left|D_{s} u\right|\right) \frac{D_{s} u}{\left|D_{s} u\right|} D_{s} v \mathrm{~d} \mu
$$

for any $v \in W_{\text {reg }}^{s, G}(\Omega)$. 


\subsection{Integration by parts formula}

Here we prove an integration by parts formula in our settings which exploits the divergence form of the operator. We introduce the following notation

$$
\left\langle\left(-\Delta_{g}\right)^{s} u, v\right\rangle_{*}=\frac{1}{2} \int_{\mathbb{R}^{2 n} \backslash\left(\Omega^{c}\right)^{2}} g\left(\left|D_{s} u\right|\right) \frac{D_{s} u}{\left|D_{s} u\right|} D_{s} v \mathrm{~d} \mu
$$

the modular

$$
\Phi_{s, G, *}(u)=\iint_{\mathbb{R}^{2 n} \backslash\left(\Omega^{c}\right)^{2}} G\left(\left|D_{s} u(x, y)\right|\right) \mathrm{d} \mu,
$$

and the corresponding Luxemburg semi-norm

$$
[u]_{W_{*}^{s, G}\left(\mathbb{R}^{n}\right)}=\inf \left\{\lambda>0: \Phi_{s, G, *}\left(\frac{u}{\lambda}\right) \leq 1\right\} .
$$

Of course, it is naturally defined the space

$$
W_{*}^{s, G}(\Omega):=\left\{u \in L^{G}(\Omega): \Phi_{s, G, *}(u)<\infty\right\} .
$$

Proposition 2.6. Given $u \in \mathcal{X}$, the following holds.

(i) The following version of the divergence theorem is true

$$
\int_{\Omega}\left(-\Delta_{g}\right)^{s} u=-\int_{\mathbb{R}^{n} \backslash \Omega} \mathcal{N}_{g} u
$$

(ii) More generally, we have the following integration by parts formula

$$
\left\langle\left(-\Delta_{g}\right)^{s} u, v\right\rangle_{*}=\int_{\Omega} v\left(-\Delta_{g}\right)^{s} u \mathrm{~d} x+\int_{\mathbb{R}^{n} \backslash \Omega} v \mathcal{N}_{g} u \mathrm{~d} x \quad \forall v \in \mathcal{X} .
$$

Proof. In light of ([16], Prop. 2.9), it suffices to prove the result for $u \in C_{c}^{2}\left(\mathbb{R}^{n}\right)$.

Let us prove $(i)$. Observe that, since the role of $x$ and $y$ are symmetric, we get

$$
\int_{\Omega} \int_{\Omega} g\left(\left|D_{s} u\right|\right) \frac{u(x)}{\left|D_{s} u\right|} \frac{\mathrm{d} x \mathrm{~d} y}{|x-y|^{n+s}}=\int_{\Omega} \int_{\Omega} g\left(\left|D_{s} u\right|\right) \frac{u(y)}{\left|D_{s} u\right|} \frac{\mathrm{d} x \mathrm{~d} y}{|x-y|^{n+s}},
$$

from where it is immediate that

$$
\int_{\Omega} \int_{\Omega} g\left(\left|D_{s} u\right|\right) \frac{D_{s} u}{\left|D_{s} u\right|} \frac{\mathrm{d} x \mathrm{~d} y}{|x-y|^{n+s}}=0
$$

Hence, we have that

$$
\begin{aligned}
\int_{\Omega}\left(-\Delta_{g}\right)^{s} u(x) \mathrm{d} x & =\int_{\Omega} \int_{\mathbb{R}^{n}} g\left(\left|D_{s} u\right|\right) \frac{D_{s} u}{\left|D_{s} u\right|} \frac{\mathrm{d} y \mathrm{~d} x}{|x-y|^{n+s}} \\
& =\int_{\Omega} \int_{\mathbb{R}^{n} \backslash \Omega} g\left(\left|D_{s} u\right|\right) \frac{D_{s} u}{\left|D_{s} u\right|} \frac{\mathrm{d} y \mathrm{~d} x}{|x-y|^{n+s}}
\end{aligned}
$$




$$
\begin{aligned}
& =\int_{\mathbb{R}^{n} \backslash \Omega}\left(\int_{\Omega} g\left(\left|D_{s} u\right|\right) \frac{D_{s} u}{\left|D_{s} u\right|} \frac{\mathrm{d} x}{|x-y|^{n+s}}\right) \mathrm{d} y \\
& =-\int_{\mathbb{R}^{n} \backslash \Omega} \mathcal{N}_{g} u(y) \mathrm{d} y,
\end{aligned}
$$

as desired. Now, let us prove (ii). Since $\mathbb{R}^{2 n} \backslash\left(\Omega^{c}\right)^{2}=\left(\Omega \times \mathbb{R}^{n}\right) \cup\left[\left(\mathbb{R}^{n} \backslash \Omega\right) \times \Omega\right]$, we get

$$
\begin{aligned}
\left\langle\left(-\Delta_{g}\right)^{s} u, v\right\rangle_{*}= & \int_{\Omega} v(x)\left(\int_{\mathbb{R}^{n}} g\left(\left|D_{s} u\right|\right) \frac{D_{s} u}{\left|D_{s} u\right|} \frac{\mathrm{d} y}{|x-y|^{n+s}}\right) \mathrm{d} x \\
& +\int_{\mathbb{R}^{n} \backslash \Omega} v(x)\left(\int_{\Omega} g\left(\left|D_{s} u\right|\right) \frac{D_{s} u}{\left|D_{s} u\right|} \frac{\mathrm{d} y}{|x-y|^{n+s}}\right) \mathrm{d} x .
\end{aligned}
$$

In light of (1.1) and (1.3) we obtain the desired relation.

Remark 2.7. If we consider the function $w_{s, \Omega}(x)=\int_{\Omega} \int_{\mathbb{R}^{n} \backslash \Omega} g\left(|x-y|^{-s}\right)|x-y|^{n+s} \mathrm{~d} y$ and the normalization of $\mathcal{N}_{g}$ given by $\tilde{\mathcal{N}}_{g}(x):=\frac{\mathcal{N}_{g}(x)}{w_{s, \Omega}(x)}$, if $\tilde{\mathcal{N}}_{g}(x)=1$ for any $x \in \mathbb{R}^{n} \backslash \bar{\Omega}$, we can define a generalization of the fractional perimeter defined in [11] as follows

$$
\int_{\mathbb{R}^{n} \backslash \Omega} \mathcal{N}_{g} \mathrm{~d} x=\int_{\mathbb{R}^{n} \backslash \Omega} w_{s, \Omega} \mathrm{d} x=\int_{\Omega} \int_{\mathbb{R}^{n} \backslash \Omega} g\left(\frac{1}{|x-y|^{s}}\right) \frac{\mathrm{d} x \mathrm{~d} y}{|x-y|^{n+s}}:=\operatorname{Per}_{s, g}(\Omega) .
$$

\section{VARIATIONAL SETting AND PROOFs OF TheOREMS 1.1 AND 1.2}

We start defining the notion of weak solution for problem (1.2). With that end it will be useful introducing the following functional settings. Let us denote

$$
\mathcal{X}:=\left\{u: \mathbb{R}^{n} \rightarrow \mathbb{R} \text { measurable s.t.: }\|u\|_{\mathcal{X}}<\infty\right\},
$$

where

$$
\|u\|_{\mathcal{X}}:=[u]_{W_{*}^{s, G}\left(\mathbb{R}^{n}\right)}+\|u\|_{L^{G}(\Omega)}+\|u\|_{L^{G, \beta}\left(\Omega^{c}\right)},
$$

and

$$
\|u\|_{L^{G, \beta}\left(\Omega^{c}\right)}=\inf \left\{\lambda>0: \int_{\mathbb{R}^{n} \backslash \Omega} \beta G\left(\frac{u}{\lambda}\right) \mathrm{d} \mu \leq 1\right\} .
$$

By following standard arguments it can be seen that $\mathcal{X}$ is a reflexive Banach space with respect to the norm $\|\cdot\|_{\mathcal{X}}$. See for instance $[16]$.

The integration by parts formula given in Proposition 2.6 leads to the following definition.

Definition 3.1. We say that $u \in \mathcal{X}$ is a weak solution of (1.2) if

$$
\left\langle\left(-\Delta_{g}\right)^{s} u, v\right\rangle_{*}+\int_{\Omega} g(|u|) \frac{u}{|u|} v \mathrm{~d} x=\lambda \int_{\Omega} f v \mathrm{~d} x+\mu \int_{\Omega} h v \mathrm{~d} x-\int_{\mathbb{R}^{n} \backslash \Omega} \beta g(|u|) \frac{u}{|u|} v \mathrm{~d} x,
$$

for all $v \in \mathcal{X}$. 
As anticipated in the introduction, we will approach problem (1.2) through the machinery of variational methods, and in particular, it will be done by using the abstract multiplicity result given in Theorem A.2. With that aim, we consider the functional $\Psi: \mathcal{X} \rightarrow \mathbb{R}$ defined as

$$
\Psi(u):=\mathcal{J}(u)-\lambda \mathcal{F}(u)-\mu \mathcal{H}(u),
$$

for every $u \in \mathcal{X}$, where $\mathcal{J}, \mathcal{F}, \mathcal{H}: \mathcal{X} \rightarrow \mathbb{R}$ are defined as

$$
\begin{gathered}
\mathcal{J}(u):=\int_{\mathbb{R}^{2 n} \backslash\left(\Omega^{c}\right)^{2}} G\left(\left|D_{s} u\right|\right) \mathrm{d} \mu+\int_{\Omega} G(|u|) \mathrm{d} x+\int_{\mathbb{R}^{n} \backslash \Omega} \beta G(|u|) \mathrm{d} x, \\
\mathcal{F}(u)=\int_{\Omega} F(x, u) \mathrm{d} x \text { and } \mathcal{H}(u)=\int_{\Omega} H(x, u) \mathrm{d} x .
\end{gathered}
$$

The following compact embedding for the space $\mathcal{X}$ holds.

Lemma 3.2. Given a Young function $A$ such that $A \prec \prec G_{*}$, then the embedding $\mathcal{X} \hookrightarrow L^{A}(\Omega)$ is compact.

Proof. Let $u \in \mathcal{X}$. Observe that $[u]_{W_{r e g}^{s, G}(\Omega)} \leq[u]_{W_{*}^{s, G}\left(\mathbb{R}^{n}\right)}$ as a consequence of the inequality

$$
\int_{\Omega \times \Omega} G\left(\frac{\left|D_{s} u\right|}{\|u\|_{*}}\right) \mathrm{d} \mu \leq \int_{\mathbb{R}^{2 n} \backslash\left(\Omega^{c}\right)^{2}} G\left(\frac{\left|D_{s} u\right|}{\|u\|_{*}}\right) \mathrm{d} \mu \leq 1,
$$

together with the definition of the Luxemburg norm. Then, from Theorem 2.4, there exists a constant $c>0$ such that

$$
\|u\|_{L^{A}(\Omega)} \leq c\left[u\left\|_{W_{r e g}^{s, G}(\Omega)} \leq c\left([u]_{W_{*}^{s, G}\left(\mathbb{R}^{n}\right)}+\|u\|_{L^{G}(\Omega)}\right) \leq c\right\| u \|_{\mathcal{X}}\right.
$$

concluding the proof due to the compactness of $W_{\text {reg }}^{s, G}(\Omega)$ into $L^{A}(\Omega)$.

The next proposition proves the well-posedness of $\Psi$.

Proposition 3.3. Let $f, h \in \mathcal{A}$, then the functional $\Psi$ is well defined on the space $\mathcal{X}$.

Proof. First, we notice that given $u \in \mathcal{X}$, from Lemma 2.5 it follows that $\mathcal{J}(u) \leq C \xi^{+}\left(\|u\|_{\mathcal{X}}\right)$ for some constant $C=C\left(p^{ \pm}\right)$. Moreover, by $\left(f_{1}\right)$ and the fact that $m$ is increasing we get

$$
\int_{\Omega} F(x, u) \mathrm{d} x \leq \int_{\Omega} w(x) \int_{0}^{u} m(|t|) \mathrm{d} t \mathrm{~d} x \leq\|w\|_{\infty} \int_{\Omega}|u| m(|u|) \mathrm{d} x .
$$

In light of Lemma 2.2, $m(|u|) \in L^{\tilde{M}}(\Omega)$, and then, by applying Hölder's inequality for Young function we get that

$$
\int_{\Omega}|u| m(|u|) \mathrm{d} x \leq\|u\|_{L^{M}(\Omega)}\|m(u)\|_{L^{\tilde{M}}(\Omega)} .
$$

Observe that ([25], Thm. 3.17.1) and Lemma 2.2 give that $\|m(u)\|_{L^{\tilde{M}}(\Omega)} \leq c\|u\|_{L^{M}(\Omega)}$. Moreover, from Lemma 3.2 it follows that $\|u\|_{L^{M}(\Omega)} \leq c\|u\|_{\mathcal{X}}$, and therefore $\mathcal{F}$ is well defined.

The well-posedness of $\mathcal{H}$ follows analogously, concluding that $\Psi$ is well defined on $\mathcal{X}$.

Next, we prove some useful properties of the functional $\mathcal{J}$. 
Lemma 3.4. Assume that $\left(G_{1}\right),\left(G_{2}\right)$ and $\left(G_{3}\right)$ hold. Then,

(i) the functional $\mathcal{J}: \mathcal{X} \rightarrow \mathbb{R}$ is $C^{1}$ with derivative given by

$$
\left\langle\mathcal{J}^{\prime}(u), v\right\rangle=\left\langle\left(-\Delta_{g}\right)^{s} u, v\right\rangle_{*}+\int_{\Omega} g(|u(x)|) \frac{u}{|u|} v(x) \mathrm{d} x+\int_{\mathbb{R}^{n} \backslash \Omega} \beta g(|u(x)|) v(x) \mathrm{d} x
$$

for all $u, v \in \mathcal{X}$

(ii) $\mathcal{J}$ is coercive, sequentially weakly lower semicontinuous;

(iii) $\mathcal{J} \in \mathcal{W}_{\mathcal{X}}$, where the class $\mathcal{W}_{\mathcal{X}}$ is given in Definition (A.1);

(iv) $\mathcal{J}$ is bounded on each bounded subset of $\mathcal{X}$ and its derivative admits a continuous inverse on $\mathcal{X}^{*}$.

Proof. (i) From ([35], Prop. 4.1), it is easy to see that $\mathcal{J}$ is class $C^{1}$.

(ii) Let $u \in \mathcal{X}$ with $\|u\|_{\mathcal{X}}>1$. In view of Lemma $2.5, \mathcal{J}$ is coercive since

$$
\mathcal{J}(u) \geq \xi^{-}\left(\|u\|_{L^{G}(\Omega)}\right)+\xi^{-}\left([u]_{W_{*}^{s, G}\left(\mathbb{R}^{n}\right)}\right)+\xi^{-}\left(\|u\|_{L^{G, \beta}\left(\Omega^{c}\right)}\right) \geq c \xi^{-}\left(\|u\|_{\mathcal{X}}\right)
$$

where $c>0$ depends only on $p^{ \pm}$. Moreover, the sequential weak lower semicontinuity of $\mathcal{J}$ follows by ([8], Lem. 19).

(iii) Let $\left\{u_{k}\right\}_{k \in \mathbb{N}}$ be a sequence in $\mathcal{X}$ such that $u_{k} \rightarrow u$ in $\mathcal{X}$ and $\liminf _{k \rightarrow \infty} \mathcal{J}\left(u_{k}\right) \leq \mathcal{J}(u)$. Then, by the sequential weak lower semicontinuity of $\mathcal{J}$ proven in (ii) we get that, up to a subsequence, $\mathcal{J}\left(u_{k}\right) \rightarrow \mathcal{J}(u)$ as $k \rightarrow+\infty$. Since $\frac{u_{k}+u}{2}$ converges weakly to $u$, and modulars are lower semicontinuous with respect to the weak convergence, we get

$$
\mathcal{J}(u) \leq \liminf _{k \rightarrow \infty} \mathcal{J}\left(\frac{u_{k}+u}{2}\right)
$$

We assume by contradiction that $u_{k}$ does not converge to $u$ in $\mathcal{X}$. Hence, there exists $\varepsilon>0$ such that $\left\|\frac{u_{k}+u}{2}\right\|_{\mathcal{X}}>$ $\varepsilon$. Then, by Lemma 2.5

$$
\mathcal{J}\left(\frac{u_{k}+u}{2}\right)>\xi^{-}(\varepsilon)
$$

On the other hand, by applying Lemma 2.3 it follows that

$$
\frac{1}{2}\left(G(u)+G\left(u_{k}\right)\right)-G\left(\frac{u_{k}+u}{2}\right) \geq G\left(\frac{u_{k}-u}{2}\right),
$$

which together with (3.2) leads to

$$
\frac{1}{2}\left(\mathcal{J}(u)+\mathcal{J}\left(u_{k}\right)\right)-\mathcal{J}\left(\frac{u_{k}+u}{2}\right) \geq \mathcal{J}\left(\frac{u_{k}-u}{2}\right)>\xi^{-}(\varepsilon) .
$$

Taking limsup in the above inequality we obtain that

$$
\mathcal{J}(u)-\xi^{-}(\varepsilon) \geq \limsup _{k \rightarrow+\infty} \mathcal{J}\left(\frac{u_{k}+u}{2}\right)
$$

which contradicts (3.1). Therefore $u_{k} \rightarrow u$ strongly in $\mathcal{X}$, and then $\mathcal{J} \in \mathcal{W}_{\mathcal{X}}$. 
(iv) When $\|u\|_{\mathcal{X}} \leq \rho$, in light of Lemma 2.5 we have that $\mathcal{J}(u) \leq \xi^{+}(\rho)$, i.e., $\mathcal{J}$ is bounded on any bounded subset of $\mathcal{X}$.

We prove now that $\mathcal{J}$ admits a continuous inverse $\mathcal{J}^{-1}: \mathcal{X}^{*} \rightarrow \mathcal{X}$ by means of the monotone operator method introduced by Browder and Minty (see ([39], Thm. 26.A (d))). Therefore, it suffices to verify that $\mathcal{J}^{\prime}$ is coercive, hemicontinuous and uniformly monotone.

Observe that since $G$ is convex, $\mathcal{J}$ also is convex. Thus $\mathcal{J}(u) \leq\left\langle\mathcal{J}^{\prime}(u), u\right\rangle$ for all $u \in \mathcal{X}$, and, by using Lemma 2.5 , for any $u \in \mathcal{X}$ such that $\|u\|_{\mathcal{X}}>1$ we have

$$
\frac{\left\langle\mathcal{J}^{\prime}(u), u\right\rangle}{\|u\|_{\mathcal{X}}} \geq \frac{\mathcal{J}(u)}{\|u\|_{\mathcal{X}}} \geq \min \left\{\|u\|_{\mathcal{X}}^{p^{-}-1},\|u\|_{\mathcal{X}}^{p^{+}-1}\right\}
$$

from where the coercivity of $\mathcal{J}^{\prime}$ follows by taking $\|u\|_{\mathcal{X}} \rightarrow \infty$.

Furthermore, since the real function $t \mapsto\left\langle\mathcal{J}^{\prime}(u+t v), w\right\rangle$ is continuous in $[0,1]$ for any $u, v, w \in \mathcal{X}$, we have that $\mathcal{J}^{\prime}$ is hemicontinuous.

Let us finally prove that $\mathcal{J}^{\prime}$ is uniformly monotone. Since $G$ is convex we have that for every $u, v \in \mathcal{X}$ it holds

$$
G(|u|) \leq G\left(\left|\frac{u+v}{2}\right|\right)+g(|u|) \frac{u}{|u|} \frac{u-v}{2} \quad \text { and } \quad G(|v|) \leq G\left(\left|\frac{u+v}{2}\right|\right)+g(|v|) \frac{v}{|v|} \frac{v-u}{2} .
$$

Adding the above two relations and integrating over $\Omega$ we find that

$$
\begin{aligned}
& \frac{1}{2} \int_{\Omega}\left(g(|u|) \frac{u}{|u|}-g(|v|) \frac{v}{|v|}\right)(u-v) \mathrm{d} x \\
& \quad \geq \int_{\Omega} G(|u|) \mathrm{d} x+\int_{\Omega} G(|v|) \mathrm{d} x-2 \int_{\Omega} G\left(\left|\frac{u+v}{2}\right|\right) \mathrm{d} x \quad \forall u, v \in \mathcal{X} .
\end{aligned}
$$

On the other hand, we deduce by Lemma 2.3 that

$$
\int_{\Omega}(G(|u|)+G(|v|)) \mathrm{d} x \geq 2 \int_{\Omega} G\left(\frac{u+v}{2}\right) \mathrm{d} x+2 \int_{\Omega} G\left(\frac{u-v}{2}\right) \mathrm{d} x \quad \forall u, v \in \mathcal{X} .
$$

From the last two relations it follows that

$$
\int_{\Omega}\left(g(|u|) \frac{u}{|u|}-g(|v|) \frac{v}{|v|}\right)(u-v) \mathrm{d} x \geq 4 \int_{\Omega} G\left(\frac{|u-v|}{2}\right) \mathrm{d} x \quad \forall u, v \in \mathcal{X} .
$$

Similarly, for any $u, v \in \mathcal{X}$ it holds that

$$
\int_{\mathbb{R}^{n} \backslash \Omega} \beta\left(g(|u|) \frac{u}{|u|}-g(|v|) \frac{v}{|v|}\right)(u-v) \mathrm{d} x \geq 4 \int_{\mathbb{R}^{n} \backslash \Omega} \beta G\left(\frac{|u-v|}{2}\right) \mathrm{d} x,
$$

and

$$
\left\langle\left(-\Delta_{g}\right)^{s}(u-v), u-v\right\rangle_{*} \geq 4 \int_{\mathbb{R}^{2 n} \backslash\left(\Omega^{c}\right)^{2}} G\left(\frac{\left|D_{s} u-D_{s} v\right|}{2}\right) \mathrm{d} \mu .
$$

Gathering the last three inequalities one gets that

$$
\left\langle\mathcal{J}^{\prime}(u)-\mathcal{J}^{\prime}(v), u-v\right\rangle \geq 4 \mathcal{J}\left(\frac{u-v}{2}\right) \quad \forall u, v \in \mathcal{X} .
$$


Define now the function $\alpha:[0,+\infty) \rightarrow[0,+\infty)$ by

$$
\alpha(t)=\frac{1}{p^{+}-2} \begin{cases}t^{p^{+}-1} & \text { for } t \leq 1 \\ t^{p^{-}-1} & \text { for } t \geq 1 .\end{cases}
$$

It is easy to check that $\alpha$ is an increasing function with $\alpha(0)=0$ and $\alpha(t) \rightarrow \infty$ as $t \rightarrow \infty$. Taking into account the above information and Lemma 2.5, we deduce that

$$
\left\langle\mathcal{J}^{\prime}(u)-\mathcal{J}^{\prime}(v), u-v\right\rangle \geq \alpha\left(\|u-v\|_{\mathcal{X}}\right)
$$

that is, $\mathcal{J}^{\prime}$ is uniformly monotone, which concludes our proof.

Lemma 3.5. $\mathcal{F}: \mathcal{X} \rightarrow \mathbb{R}$ is $C^{1}$ with derivative given by

$$
\left\langle\mathcal{F}^{\prime}(u), v\right\rangle=\int_{\Omega} f(x, u) v \mathrm{~d} x
$$

for all $u, v \in \mathcal{X}$. Moreover, $\mathcal{F}: \mathcal{X} \rightarrow \mathcal{X}^{*}$ is compact.

Proof. Usual arguments show that $\mathcal{F} \in C^{1}(\mathcal{X}, \mathbb{R})$. In order to verify the compactness of $\mathcal{F}$, let $\left\{u_{k}\right\}_{k \in \mathbb{N}} \subset \mathcal{X}$ be a bounded sequence. Then up to a subsequence $u_{k}$ weakly converges in $\mathcal{X}$ to $u \in \mathcal{X}$. Moreover, in light of Lemma $3.2, u_{k} \rightarrow u$ strongly in $L^{M}(\Omega)$ and a.e. in $\Omega$.

Fixed $v \in \mathcal{X}$ with $\|v\|_{\mathcal{X}} \leq 1$, thanks to the Hölder's inequality for Young functions and the embedding of Lemma 3.2 we have

$$
\begin{aligned}
\left|\left\langle\mathcal{F}^{\prime}\left(u_{k}\right), v\right\rangle-\left\langle\mathcal{F}^{\prime}(u), v\right\rangle\right| & =\left|\int_{\Omega}\left(f\left(x, u_{k}\right)-f(x, u)\right) v \mathrm{~d} x\right| \\
& \leq\left\|f\left(\cdot, u_{k}(\cdot)\right)-f(\cdot, u(\cdot))\right\|_{L^{\tilde{M}}(\Omega)}\|v\|_{L^{M}(\Omega)} \\
& \leq c\left\|f\left(\cdot, u_{k}(\cdot)\right)-f(\cdot, u(\cdot))\right\|_{L^{\tilde{M}}(\Omega)}\|v\|_{\mathcal{X}},
\end{aligned}
$$

for some $c>0$. Thus, taking supremum for $\|v\|_{\mathcal{X}} \leq 1$, we get

$$
\left\|\mathcal{F}^{\prime}\left(u_{k}\right)-\mathcal{F}^{\prime}(u)\right\|_{\mathcal{X}^{*}} \leq c\left\|f\left(\cdot, u_{k}(\cdot)\right)-f(\cdot, u(\cdot))\right\|_{L^{\tilde{M}}(\Omega)} .
$$

Being $f \in \mathcal{A}$ we deduce immediately that

$$
f\left(x, u_{k}(x)\right)-f(x, u(x)) \rightarrow 0 \text { as } k \rightarrow \infty,
$$

for almost all $x \in \Omega$ and

$$
\begin{aligned}
\left|f\left(x, u_{k}(x)\right)-f(x, u(x))\right| & \leq\left|f\left(x, u_{k}(x)\right)\right|+|f(x, u(x))| \\
& \leq\|w\|_{\infty}\left(m\left(\left|u_{k}(x)\right|\right)+m(|u(x)|)\right) .
\end{aligned}
$$

Note that the majorant function in the previous relation is uniformly bounded in $L^{\tilde{M}}(\Omega)$. Hence, by applying the dominate convergence theorem we get that

$$
\int_{\Omega} \tilde{M}\left(\left|f\left(x, u_{k}(x)\right)-f(x, u(x))\right|\right) \mathrm{d} x \rightarrow 0 \quad \text { as } k \rightarrow \infty .
$$


Since $M$ satisfies $\left(G_{1}\right), \tilde{M}$-mean convergence is equivalent to norm convergence (see [25], Lem. 3.10.4), that is,

$$
\left\|f\left(\cdot, u_{k}(\cdot)\right)-f(\cdot, u(\cdot))\right\|_{L^{\tilde{M}}(\Omega)} \rightarrow 0 \quad \text { as } k \rightarrow \infty .
$$

Therefore $\left\|\mathcal{F}^{\prime}\left(u_{k}\right)-\mathcal{F}^{\prime}(u)\right\|_{\mathcal{X}^{*}} \rightarrow 0$ as $k \rightarrow \infty$, giving that $\mathcal{F}^{\prime}$ is a compact operator.

Remark 3.6. Combining Lemmas 3.4 and 3.5, we deduce that $\Psi \in C^{1}(\mathcal{X}, \mathbb{R})$ with the derivative given by

$$
\begin{aligned}
\left\langle\Psi^{\prime}(u), v\right\rangle= & \left\langle\left(-\Delta_{g}\right)^{s} u, v\right\rangle_{*}+\int_{\mathbb{R}^{n} \backslash \Omega} \beta g(|u(x)|) \frac{u}{|u|} v(x) \mathrm{d} x+\int_{\Omega} g(|u(x)|) \frac{u}{|u|} v(x) \mathrm{d} x \\
& -\lambda \int_{\Omega} f(x, u(x)) v(x) \mathrm{d} x-\mu \int_{\Omega} g(x, u(x)) v(x) \mathrm{d} x,
\end{aligned}
$$

for every $v \in \mathcal{X}$. Then, critical points of $\Psi$ are weak solutions of problem (1.2).

Having proved these preliminaries, we are in position to prove our first main theorem.

Proof of Theorem 1.1. Fix $\lambda, \mu$ and $f, h \in \mathcal{A}$, we check the conditions needed to apply Theorem A.2.

Fixed $\varepsilon>0$, in light of $\left(F_{1}\right)$ there exist intervals $I_{1}=\left[-r_{2},-r_{1}\right]$ and $I_{2}=\left[r_{1}, r_{2}\right]$ such that

$$
F(x, t) \leq \varepsilon G(|t|) \quad(x, t) \in \Omega \times \mathbb{R} \backslash\left(I_{1} \cup I_{2}\right) .
$$

In $I_{1} \cup I_{2}, F(x, \cdot)$ is bounded in $\Omega$, then there exist $\mathrm{d}>0$ and a Young function $B$ such that $b=B^{\prime}, G \prec \prec$ $B \prec \prec G_{*}$ and $p^{+}<b^{-}$(here $b^{-}$denotes a constant such that $b^{-}<\frac{t b(t)}{B(t)}$ ) for which

$$
F(x, t) \leq \mathrm{d} B(|t|) \quad(x, t) \in \Omega \times\left(I_{1} \cup I_{2}\right) .
$$

Then, from the inequalities above we obtain that

$$
\frac{\mathcal{F}(u)}{\mathcal{J}(u)} \leq \frac{\int_{\Omega} F(x, u) \mathrm{d} x}{\int_{\Omega} G(|u|) \mathrm{d} x} \leq \varepsilon+\mathrm{d} \frac{\int_{\Omega} B(|u|) \mathrm{d} x}{\int_{\Omega} G(|u|) \mathrm{d} x} .
$$

Observe that, assuming that $\|u\|_{\mathcal{X}} \leq 1$, from Lemma 2.5 and ([25], Thm 3.17.1) it holds that

$$
\lim _{u \rightarrow 0} \frac{\int_{\Omega} B(|u|) \mathrm{d} x}{\int_{\Omega} G(|u|) \mathrm{d} x} \leq \lim _{u \rightarrow 0} \frac{\|u\|_{L^{B}(\Omega)}^{b^{-}}}{\|u\|_{L^{G}(\Omega)}^{p^{+}}} \leq c \lim _{u \rightarrow 0}\|u\|_{L^{G}(\Omega)}^{b^{-}-p^{+}}=0 .
$$

From the previous computations it follows that

$$
J_{1}:=\limsup _{u \rightarrow 0} \frac{\mathcal{F}(u)}{\mathcal{J}(u)} \leq \varepsilon
$$

Moreover, assuming that $\|u\|_{\mathcal{X}} \geq 1$, by using again (3.3) and Lemma 2.5 we get

$$
\begin{aligned}
\frac{\mathcal{F}(u)}{\mathcal{J}(u)} & \leq \frac{\int_{\left\{x \in \Omega:|u(x)| \leq r_{2}\right\}} F(x, u) \mathrm{d} x}{\mathcal{J}(u)}+\frac{\int_{\left\{x \in \Omega:|u(x)|>r_{2}\right\}} F(x, u) \mathrm{d} x}{\int_{\Omega} G(|u|) \mathrm{d} x} \\
& \leq \frac{|\Omega|}{\|u\|_{\mathcal{X}}^{p^{+}}} \sup \left\{F(x, u(x)):(x, u(x)) \in \Omega \times\left[-r_{2}, r_{2}\right]\right\}+\varepsilon,
\end{aligned}
$$


from where we obtain that

$$
J_{2}:=\limsup _{\|u\| \rightarrow \infty} \frac{\mathcal{F}(u)}{\mathcal{J}(u)} \leq \varepsilon
$$

Therefore, since $\varepsilon$ is arbitrary we obtain that $\max \left\{0, J_{1}, J_{2}\right\}=0$.

Finally, since we are assuming $\left(F_{2}\right)$ it follows that the quantity $\sup \left\{\mathcal{F}(u) / \mathcal{J}(u): u \in \mathcal{J}^{-1}([0, \infty])\right\}$ is strictly positive.

Finally, gathering Lemmas 3.4, 3.5 and the last computations, we are in position of applying Theorem A.2 to obtain our conclusion.

Finally, we prove our second existence result.

Proof of Theorem 1.2. Since $G \prec \prec G_{*}$, hypothesis $(i)$ implies $\left(f_{1}\right)$.

Note that hypothesis $(i)$ also implies that

$$
\frac{\mathcal{F}(u)}{\mathcal{J}(u)} \leq \frac{\int_{\Omega} F(x, u) \mathrm{d} x}{\int_{\Omega} G(|u|) \mathrm{d} x} \leq c_{1} \frac{\int_{\Omega}(1+B(|u|) \mathrm{d} x)}{\int_{\Omega} G(|u|) \mathrm{d} x} .
$$

Assuming that $\|u\|_{\mathcal{X}} \geq 1$, from Lemma 2.5 and ([25], Thm. 3.17.1) it holds that

$$
\lim _{\|u\|_{\mathcal{X}} \rightarrow \infty} \frac{\int_{\Omega} B(|u|) \mathrm{d} x}{\int_{\Omega} G(|u|) \mathrm{d} x} \leq \lim _{u \rightarrow \infty} \frac{\|u\|_{L^{B}(\Omega)}^{b^{+}}}{\|u\|_{L^{G}(\Omega)}^{p^{-}}} \leq c \lim _{u \rightarrow \infty}\|u\|_{L^{G}(\Omega)}^{b^{+}-p^{-}}=0 .
$$

From where

$$
J_{1}:=\limsup _{\|u\|_{\mathcal{X}} \rightarrow \infty} \frac{\mathcal{F}(u)}{\mathcal{J}(u)}=0
$$

Similarly, assuming that $\|u\|_{\mathcal{X}} \leq 1$, hypothesis (ii) implies that

$$
J_{2}:=\limsup _{\|u\|_{\mathcal{X}} \rightarrow 0} \frac{\mathcal{F}(u)}{\mathcal{J}(u)} \leq c_{2} \lim _{\|u\|_{\mathcal{X}} \rightarrow 0} \frac{\int_{\Omega} D(|u|) \mathrm{d} x}{\int_{\Omega} G(|u|) \mathrm{d} x} \leq c \lim _{\|u\|_{\mathcal{X}} \rightarrow 0}\|u\|_{L^{G}(\Omega)}^{\mathrm{d}^{-}-p^{+}}=0 .
$$

From these relations it follows that $\max \left\{0, J_{1}, J_{2}\right\}=0$.

Now, without loss of generality we assume that $\tau_{2}>0$ and choose a function $u \in \mathcal{X}$ such that $u(x) \geq 0$ in $\Omega$ and such that there exists $x_{0} \in \Omega$ with $u\left(x_{0}\right)>\tau_{2}$. It follows that $\mathcal{U}:=\left\{x \in \Omega: u(x)>\tau_{2}\right\}$ is a nonempty open subset of $\Omega$.

Let $k: \mathbb{R} \rightarrow \mathbb{R}$ defined by $k(t)=\min \left\{t, \tau_{2}\right\}$. Then $k(0)=0$ and $k$ is Lipschitz with Lipschitz constant 1 . Therefore, the function $u_{1}=k \circ u \in \mathcal{X}$ satisfies that $u_{1}(x)=t$ for every $x \in U$ and $0 \leq u_{1}(x) \leq \tau_{2}$ for every $x \in \Omega$. Then, by hypothesis (iii) we obtain that

$$
F\left(x, u_{1}(x)\right)>0 \quad \text { for any } x \in \mathcal{U}, \quad F\left(x, u_{1}(x)\right) \geq 0 \quad \text { for every } x \in \Omega .
$$

From this we conclude that $\mathcal{F}\left(u_{1}\right)>0$ and thus

$$
\delta^{-1}=\sup \left\{\frac{\mathcal{F}(u)}{\mathcal{J}(u)}: u \in \mathcal{J}^{-1}((0, \infty))\right\}>0 .
$$


Therefore, from Lemma 3.4, Lemma 3.5 and the last computations, the result follows by applying Theorem A.2.

\section{SOME EXAMPLES OF NONLINEARITIES}

Let $G$ be a Young function satisfying $\left(G_{1}\right),\left(G_{2}\right)$ and $\left(G_{3}\right)$. Let us prove that the following examples of nonlinearities belong to the class $\mathcal{A}$ and satisfy the hypothesis of Theorem 1.1.

(i) Consider the function $f(t)=p|\sin t|^{p-2} \sin t \cos t$ with $p^{+}<p<p_{*}^{+}$and observe that

$$
|f(t)| \leq p\left(1+|t|^{p_{*}^{+}-1}\right),
$$

and since $F(t)=|\sin t|^{p}$ we obtain

$$
\lim _{|t| \rightarrow 0} \frac{\sup _{x} F(t)}{G(t)} \leq \lim _{|t| \rightarrow 0} \frac{|\sin t|^{p}}{|t|^{p^{+}}}=0, \quad \lim _{|t| \rightarrow \infty} \frac{\sup _{x} F(t)}{G(t)} \leq \lim _{|t| \rightarrow \infty} \frac{|\sin t|^{p}}{|t|^{p^{-}}}=0 .
$$

Finally, given a compact set $C \subset \Omega$ of positive measure, we consider a function $v \in \mathcal{X}$ such that $v(x)=\frac{\pi}{2}$ in $C$ and $0 \leq v(x) \leq \frac{\pi}{2}$ in $\Omega \backslash C$. Then

$$
\sup _{u \in \mathcal{X}} \int_{\Omega} F(u) \mathrm{d} x \geq \int_{\Omega}|\sin v(x)|^{p} \mathrm{~d} x=|C|+\int_{\Omega \backslash C}|\sin v(x)|^{p} \mathrm{~d} x>0 .
$$

(ii) More generally, let $M$ be a Young function such that $p^{-}<p^{+}<m^{-}<m^{+}$, where

$$
1<m^{-}<\frac{t m(t)}{M(t)}<m^{+}<\infty \quad \text { for all } t \geq 0
$$

Consider the function $f(t)=m(|\sin t|) \cos t$ for $t \geq 0$, and observe that this function fulfills that $|f(t)| \leq$ $\max \{m(1), 1\}+m(|t|)$. Moreover, taking $\tau=\sin r$, we get

$$
\int_{0}^{t} m(\sin r) \cos r \mathrm{~d} r=\int_{0}^{t} m(\tau) \mathrm{d} \tau=M(|\sin t|),
$$

from where

$$
\lim _{|t| \rightarrow 0} \frac{\sup _{x} F(t)}{G(t)} \leq \lim _{|t| \rightarrow 0} \frac{M(|\sin t|)}{G(|t|)}=0 \leq \lim _{|t| \rightarrow 0} \frac{|\sin t|^{m^{-}}}{|t|^{p^{+}}}=0
$$

and

$$
\lim _{|t| \rightarrow \infty} \frac{\sup _{x} F(t)}{G(t)} \leq \lim _{|t| \rightarrow \infty} \frac{M(|\sin t|)}{G(|t|)}=0 \leq \lim _{|t| \rightarrow \infty} \frac{|\sin t|^{m^{+}}}{|t|^{p^{-}}}=0 .
$$

As before, given a compact set $C \subset \Omega$ of positive measure, we consider a function $u \in \mathcal{X}$ such that $v(x)=\frac{\pi}{2}$ in $C$ and $0 \leq v(x) \leq \frac{\pi}{2}$ in $\Omega \backslash C$. Then

$$
\sup _{u \in \mathcal{X}} \int_{\Omega} F(u) \mathrm{d} x \geq \int_{\Omega} G(|\sin v(x)|) \mathrm{d} x=|C|+\int_{\Omega \backslash C} G(|\sin v(x)|) \mathrm{d} x>0 .
$$


(iii) We consider the following concave-convex combination

$$
f(t)=t^{p-1}-t^{q-1} \quad \text { with } p^{+}<p<q<p_{*}^{+}:=\frac{n p^{+}}{n-s p^{+}} .
$$

Note that for some positive constant $c=c\left(p^{ \pm}\right)$it holds that

$$
|f(t)| \leq c\left(1+|t|^{p-1}\right) \leq c\left(1+|t|^{p_{*}^{+}-1}\right)
$$

Moreover,

$$
\lim _{|t| \rightarrow 0} \frac{\sup _{x} F(t)}{G(t)} \leq \lim _{|t| \rightarrow 0} \frac{\frac{|t|^{p}}{p}-\frac{|t|^{q}}{q}}{|t|^{p^{+}}}=0, \quad \lim _{|t| \rightarrow \infty} \frac{\sup _{x} F(x, t)}{G(t)} \leq \lim _{|t| \rightarrow \infty} \frac{\frac{|t|^{p}}{p}-\frac{|t|^{q}}{q}}{|t|^{p^{-}}}=-\infty .
$$

Finally, let a compact set $C \subset \Omega$ large enough and $v \in \mathcal{X}$ such that $v(x)=\tau$ in $C$ and $0 \leq v(x) \leq \tau$ in $\Omega \backslash C$, where $\tau$ is chosen such that $\frac{\tau^{q}}{q}-\frac{\tau^{p}}{p}>0$. Then

$$
\begin{aligned}
\sup _{u \in \mathcal{X}} \int_{\Omega} F(u) \mathrm{d} x & \geq \int_{\Omega} F(v) \mathrm{d} x=\frac{1}{q} \int_{\Omega} v^{q} \mathrm{~d} x-\frac{1}{p} \int_{\Omega} v^{p} \mathrm{~d} x \\
& \geq \frac{1}{q} \int_{C} v^{q} \mathrm{~d} x-\frac{1}{p} \int_{C} v^{p} \mathrm{~d} x-\frac{1}{p} \int_{\Omega \backslash C} v^{p} \mathrm{~d} x \\
& \geq|C|\left(\frac{\tau^{q}}{q}-\frac{\tau^{p}}{p}\right)-\frac{\tau^{p}}{p}|\Omega \backslash C|>0 .
\end{aligned}
$$

The following example satisfies the hypothesis of Theorem 1.2.

(iv) Let $0<\alpha<p^{-} \leq p^{+}<\beta$. Consider the function

$$
f_{1}(t)= \begin{cases}|t|^{\alpha-2} t & \text { if }|t| \leq 1 \\ |t|^{\beta-2} t & \text { if }|t|>1\end{cases}
$$

Then, it easily follows that

$$
F_{1}(t)= \begin{cases}\frac{|t|^{\alpha}}{\alpha} & \text { if }|t| \leq 1 \\ \frac{1}{\alpha}-\frac{1}{\beta}+\frac{1}{\beta}|t|^{\beta} & \text { if }|t|>1\end{cases}
$$

and conditions $(i)-($ iii $)$ from Theorem 1.2 are fulfilled.

\section{Eigenvalues AND Minimizers}

We start this section by defining the notion of eigenvalues.

Definition 5.1. We say that $\lambda$ is an eigenvalue of (1.4) with eigenfunction $u \in W_{0}^{s, G}(\Omega)$ if

$$
\left\langle\left(-\Delta_{g}\right)^{s} u, v\right\rangle=(\lambda-1) \int_{\Omega} g(|u|) \frac{u}{|u|} v \mathrm{~d} x \quad \forall v \in W_{0}^{s, G}(\Omega) .
$$


We say that $\lambda$ is an eigenvalue of (1.5) with eigenfunction $u \in W_{*}^{s, G}(\Omega)$ if

$$
\left\langle\left(-\Delta_{g}\right)^{s} u, v\right\rangle_{*}=(\lambda-1) \int_{\Omega} g(|u|) \frac{u}{|u|} v \mathrm{~d} x \quad \forall v \in W_{*}^{s, G}(\Omega) .
$$

We say that $\lambda$ is an eigenvalue of (1.6) with eigenfunction $u \in W_{\text {reg }}^{s, G}(\Omega)$ if

$$
\left\langle\left(-\Delta_{g}\right)_{\Omega}^{s} u, v\right\rangle=(\lambda-1) \int_{\Omega} g(|u|) \frac{u}{|u|} v \mathrm{~d} x \quad \forall v \in W_{\text {reg }}^{s, G}(\Omega) .
$$

We say that $\lambda$ is an eigenvalue of (1.7) with eigenfunction $u \in \mathcal{X}$ if

$$
\left\langle\left(-\Delta_{g}\right)_{\Omega}^{s} u, v\right\rangle_{*}=(\lambda-1) \int_{\Omega} g(|u|) \frac{u}{|u|} v \mathrm{~d} x-\int_{\mathbb{R}^{n} \backslash \Omega} \beta g(|u|) \frac{u}{|u|} v \mathrm{~d} x \quad \forall v \in \mathcal{X} .
$$

In order to prove our eigenvalues and minimizers results we will consider the following functionals defined in Sections 2.2 and 2.4

$$
\Phi_{s, G, \mathbb{R}^{n}}(u): W_{0}^{s, G}(\Omega) \rightarrow \mathbb{R}, \quad \Phi_{s, G, \Omega}(u): W_{\text {reg }}^{s, G}(\Omega) \rightarrow \mathbb{R}, \quad \Phi_{s, G, *}(u): W_{*}^{s, G}(\Omega) \rightarrow \mathbb{R} .
$$

and

$$
\Phi_{G, \Omega}(u): \mathcal{W}(\Omega) \rightarrow \mathbb{R}
$$

where its definition domain $\mathcal{W}(\Omega)$ is either $W_{0}^{s, G}(\Omega), W_{\text {reg }}^{s, G}(\Omega)$ or $W_{*}^{s, G}(\Omega)$.

Following ([35], Prop. 4.1) it is straightforward to see that these functionals are well-defined and are Fréchet derivable. Moreover, the following expressions can be deduced.

Proposition 5.2. We have that $\left(\Phi_{s, G, \mathbb{R}^{n}}\right)^{\prime}$ is defined from $W_{0}^{s, G}(\Omega)$ onto its dual, $\left(\Phi_{s, G, \Omega}\right)^{\prime}$ from $W_{\text {reg }}^{s, G}(\Omega)$ onto its dual, $\left(\Phi_{s, G, *}\right)^{\prime}$ from $W_{*}^{s, G}(\Omega)$ onto its dual, and $\left(\Phi_{G, \Omega}\right)^{\prime}$ from $\mathcal{W}(\Omega)$ onto its dual, are $C^{1}$ and their Fréchet derivatives are given by

$$
\begin{array}{ll}
\left\langle\left(\Phi_{s, G, \mathbb{R}^{n}}\right)^{\prime}(u), v\right\rangle=\left\langle\left(-\Delta_{g}\right)^{s} u, v\right\rangle & \forall v \in W_{0}^{s, G}(\Omega), \\
\left\langle\left(\Phi_{s, G, \Omega}\right)^{\prime}(u), v\right\rangle=\left\langle\left(-\Delta_{g}\right)^{s} u, v\right\rangle & \forall v \in W_{r e g}^{s, G}(\Omega), \\
\left\langle\left(\Phi_{s, G, *}\right)^{\prime}(u), v\right\rangle=\left\langle\left(-\Delta_{g}\right)^{s} u, v\right\rangle_{*} & \forall v \in W_{*}^{s, G}(\Omega), \\
\left\langle\left(\Phi_{G, \Omega}\right)^{\prime}(u), v\right\rangle=\int_{\Omega} g(|u|) \frac{u}{|u|} v \mathrm{~d} x & \forall v \in \mathcal{W}(\Omega) .
\end{array}
$$

Proof. See ([35], Prop. 4.1) with the pertinent changes.

Given $\mu>0$, we consider the minimization problems

$$
\begin{gathered}
\Lambda_{D}:=\inf _{u \in M_{\mu}^{D}} \frac{\Phi_{s, G, \mathbb{R}^{n}}(u)+\Phi_{G, \Omega}(u)}{\Phi_{G, \Omega}(u)} \quad \text { with } \quad M_{\mu}^{D}=\left\{u \in W_{0}^{s, G}: \Phi_{G, \Omega}(u)=\mu\right\}, \\
\Lambda_{N}:=\inf _{u \in M_{\mu}^{N}} \frac{\Phi_{s, G, *}(u)+\Phi_{G, \Omega}(u)}{\Phi_{G, \Omega}(u)} \quad \text { with } \quad M_{\mu}^{N}=\left\{u \in W_{*}^{s, G}(\Omega): \Phi_{G, \Omega}(u)=\mu\right\},
\end{gathered}
$$




$$
\Lambda_{\tilde{N}}:=\inf _{u \in M_{\mu}^{\tilde{N}}} \frac{\Phi_{s, G, \Omega}(u)+\Phi_{G, \Omega}(u)}{\Phi_{G, \Omega}(u)} \quad \text { with } \quad M_{\mu}^{\tilde{N}}=\left\{u \in W_{r e g}^{s, G}(\Omega): \Phi_{G, \Omega}(u)=\mu\right\}
$$

and

$$
\Lambda_{R}:=\inf _{u \in M_{\mu}^{R}} \frac{\Phi_{s, G, *}(u)+\Phi_{G, \Omega}(u)+\Phi_{G, \beta, \Omega^{c}}(u)}{\Phi_{G, \Omega}(u)},
$$

with

$$
M_{\mu}^{R}=\left\{u \in \mathcal{X}: \Phi_{G, \Omega}(u)=\mu\right\} \quad \text { and } \quad \Phi_{G, \beta, \Omega^{c}}(u):=\int_{\mathbb{R}^{n} \backslash \Omega} \beta G(|u(x)|) \mathrm{d} x .
$$

Note the subindex refers to Dirichlet, Neumann, regional Neumann and Robin, respectively. Moreover, due to the possible lack of homogeneity, in general the quantities defined above depend on the energy level $\mu$.

Remark 5.3. By using the Poincaré's inequality ([35], Prop. 3.2) it follows that $[\cdot]$ is an equivalent norm in $W_{0}^{s, G}(\Omega)$.

Proposition 5.4. For each $\mu>0$ there exist solutions of the minimization problems (5.5), (5.6), (5.7) and (5.8), respectively.

Proof. It follows just by applying the direct method of the calculus of variations. (See [35], Prop. 5.1).

Existence of minimizers allow us to prove existence of eigenvalues.

Theorem 5.5. For every $\mu>0$ there exist positive numbers $\lambda_{D}, \lambda_{N}, \lambda_{\tilde{N}}$ and $\lambda_{R}$ which are eigenvalues of (1.4), (1.5), (1.6) and (1.7), respectively, with non-negative eigenfunctions $u_{D} \in W_{0}^{s, G}(\Omega), u_{N} \in W_{*}^{s, G}(\Omega), u_{\tilde{N}} \in$ $W_{\text {reg }}^{s, G}(\Omega)$ and $u_{R} \in \mathcal{X}$, respectively, normalized such that $\Phi_{G, \Omega}\left(u_{D}\right)=\Phi_{G, \Omega}\left(u_{N}\right)=\Phi_{G, \Omega}\left(u_{\tilde{N}}\right)=\Phi_{G, \Omega}\left(u_{R}\right)=\mu$.

Proof. Given a fixed $\mu>0$, in light of Proposition 5.4 there exist functions $u_{D} \in W_{0}^{s, G}(\Omega), u_{N} \in W_{*}^{s, G}\left(\mathbb{R}^{n}\right)$, $u_{\tilde{N}} \in W^{s, G}(\Omega)$ and $u_{R} \in \mathcal{X}$, respectively, normalized such that their modular $\Phi_{G, \Omega}$ is equal to $\mu$, which attain the minimization problems (5.5), (5.6), (5.7) and (5.8), respectively.

Therefore, by the Lagrange multipliers rule, since the involved functionals are $C^{1}$, there exist numbers $\lambda_{D}$, $\lambda_{N}, \lambda_{\tilde{N}}$ and $\lambda_{R}$ for which the corresponding function $u_{D}, u_{N}, u_{\tilde{N}}$ and $u_{R}$ satisfy the weak formulations (5.1), (5.2), (5.3) and (5.4), respectively.

Proposition 5.6. The following relations among the minimizers of (5.5), (5.6) and (5.7) holds

$$
\Lambda_{\tilde{N}} \leq \Lambda_{N} \leq \Lambda_{R} \leq \Lambda_{D}
$$

Proof. Observe that since $\Phi_{s, G, \Omega}(u) \leq \Phi_{s, G, *}(u)$ it follows that $W_{*}^{s, G}(\Omega) \subset W_{\text {reg }}^{s, G}(\Omega)$. Then, given a minimizer $u \in M_{\mu}^{N}$ of $\Lambda_{N}$ we get that

$$
\min _{u \in W_{*}^{s, G}(\Omega)} \frac{1}{\mu}\left(\mu+\Phi_{s, G, \Omega}(u)\right) \leq \min _{u \in W_{s, G, *}(\Omega)} \frac{1}{\mu}\left(\mu+\Phi_{*}^{s, G}(u),\right)=\Lambda_{N},
$$

but since minimizing over a small set enlarges the minimum, we conclude that

$$
\Lambda_{\tilde{N}}=\min _{u \in W_{r e g}^{s, G}(\Omega)} \frac{1}{\mu}\left(\mu+\Phi_{s, G, \Omega}(u)\right) \leq \min _{u \in W_{*}^{s, G}(\Omega)} \frac{1}{\mu}\left(\mu+\Phi_{s, G, \Omega}(u)\right) \leq \Lambda_{N} .
$$


Moreover, since $\mathcal{X} \subset W_{*}^{s, G}(\Omega)$ it follows that $\Lambda_{N} \leq \Lambda_{R}$.

Finally, note that $u \in W_{0}^{s, G}(\Omega)$ if $\Phi_{s, G, *}(u)<\infty$ and $u=0$ in $\mathbb{R}^{n} \backslash \Omega$. Therefore, $W_{0}^{s, G}(\Omega) \subset \mathcal{X}$ and $\Phi_{s, G, \mathbb{R}^{n}}(u)=\Phi_{s, G, *}(u)$. Then, proceeding as before, $\Lambda_{R} \leq \Lambda_{D}$.

The following proposition claims that minimizers are uniformly bounded away from zero independently of the energy level.

Proposition 5.7. Given $\mu>0$, the minimizers $\Lambda_{\tilde{N}}, \Lambda_{N}, \Lambda_{R}$ and $\Lambda_{D}$ are positive and bounded by below independently on $\mu$.

Proof. The Dirichlet case is treated in ([35], Thm. 4.2). We deal here with the general case.

Given $\mu>0$, let $u \in M_{\mu}^{\tilde{N}}$ be a minimizer of $\Lambda_{\tilde{N}}$, that is, $u \in W_{\text {reg }}^{s, G}(\Omega)$ is such that $\Phi_{G, \Omega}(u)=\mu$ and

$$
\Lambda_{\tilde{N}}=\frac{\Phi_{s, G, \Omega}(u)+\Phi_{G, \Omega}(u)}{\Phi_{G, \Omega}(u)} .
$$

Denote by $\bar{u}=\frac{1}{|\Omega|} \int_{\Omega} u(x) \mathrm{d} x$ the average of $u$ on $\Omega$. By using the $\Delta_{2}$ condition we have that

$$
\int_{\Omega} G(|u|) \mathrm{d} x \leq \mathbf{C} \int_{\Omega} G(|u-\bar{u}|) \mathrm{d} x+\mathbf{C} \int_{\Omega} G(|\bar{u}|) \mathrm{d} x
$$

By using Jensen's inequality and $\left(L_{1}\right)$ we get

$$
\begin{aligned}
\int_{\Omega} G(|u-\bar{u}|) \mathrm{d} x & =\int_{\Omega} G\left(\left|\frac{1}{|\Omega|} \int_{\Omega}(u(x)-u(y)) \mathrm{d} y\right|\right) \mathrm{d} x \\
& \leq \frac{1}{|\Omega|} \int_{\Omega} \int_{\Omega} G(|u(x)-u(y)|) \mathrm{d} y \mathrm{~d} x \\
& \leq \frac{1}{|\Omega|} \int_{\Omega} \int_{\Omega} G\left(\frac{|u(x)-u(y)|}{|x-y|^{s}} \operatorname{diam}(\Omega)^{s}\right) \mathrm{d} y \mathrm{~d} x \\
& \leq c(|\Omega|) \Phi_{s, G, \Omega}(u)
\end{aligned}
$$

Finally, since again the Jensen's inequality gives

$$
\int_{\Omega} G(|\bar{u}|) \leq \int_{\Omega} G\left(\frac{1}{|\Omega|} \int_{\Omega}|u(y)| \mathrm{d} y\right) \mathrm{d} x \leq \frac{1}{|\Omega|} \int_{\Omega} \int_{\Omega} G(|u(y)|) \mathrm{d} y \mathrm{~d} x=\Phi_{G, \Omega}(u)
$$

we obtain that $\Phi_{G, \Omega}(u) \leq c(\mathbf{C},|\Omega|)\left(\Phi_{s, G, \Omega}(u)+\Phi_{G, \Omega}(u)\right)$, which implies a lower bound for $\Lambda_{\tilde{N}}$ :

$$
\Lambda_{\tilde{N}}=\frac{\Phi_{s, G, \Omega}(u)+\Phi_{G, \Omega}(u)}{\Phi_{G, \Omega}(u)} \geq \frac{1}{c(|\Omega|)} .
$$

In view of Proposition 5.6, the same lower bound is admissible for $\Lambda_{N}, \Lambda_{R}$ and $\Lambda_{D}$.

The following proposition states that, although eigenvalues and minimizers differ in general, both quantities are indeed comparable.

Proposition 5.8. It holds that

$$
\frac{p^{-}}{p^{+}} \Lambda \leq \lambda \leq \frac{p^{+}}{p^{-}} \Lambda
$$


where $\Lambda \in\left\{\Lambda_{D}, \Lambda_{R}, \Lambda_{N}, \lambda_{\tilde{N}}\right\}$ and $\Lambda \in\left\{\lambda_{D}, \lambda_{R}, \lambda_{N}, \lambda_{\tilde{N}}\right\}$, respectively.

As a direct consequence, denoting $c=p^{+} / p^{-}$, we have

$$
\lambda_{\tilde{N}} \leq c^{2} \lambda_{N} \leq c^{4} \lambda_{R} \leq c^{6} \lambda_{D} .
$$

Proof. These first chain of inequalities just follow by testing in the definition of eigenvalue with the eigenfunction itself and using the fact that condition $\left(G_{1}\right)$, for all $t \geq 0$, relates $\operatorname{tg}(t)$ with $G(t)$ up to the constants $p^{ \pm}$.

The second chain of inequalities are obtained just gathering the first one together with Proposition 5.6.

As a consequence of Proposition 5.7 and 5.8 we obtain a lower bound for eigenvalues.

Theorem 5.9. $\lambda_{D}, \lambda_{R}, \lambda_{N}, \lambda_{\tilde{N}}$ are bounded by below by a positive constant independent on $\mu$.

\section{Appendix A. An Abstract existence Result}

Definition A.1. We introduce the following definitions.

(i) If $X$ is a real Banach space, we denote by $\mathcal{W}_{X}$ the class of all functionals $\mathcal{J}: X \rightarrow \mathbb{R}$ possessing the following property: if $\left\{u_{k}\right\}_{k \in \mathbb{N}}$ is a sequence in $X$ converging weakly to $u \in X$ and $\liminf _{k \rightarrow \infty} \mathcal{J}\left(u_{k}\right) \leq \mathcal{J}(u)$, then $\left\{u_{k}\right\}_{k \in \mathbb{N}}$ has a subsequence converging strongly to $u$.

(ii) We say that the derivative of $\mathcal{J}$ admits a continuous inverse on $X^{*}$ we mean that there exists a continuous operator $T: X^{*} \rightarrow X$ such that $T(\mathcal{J}(x))=x$ for all $x \in X$.

The above property is somehow a compactness property, stating the existence of a convergent subsequence of a given sequence.

Theorem A.2 ([34]). Let $X$ be a separable and reflexive real Banach space; $\mathcal{J}: X \rightarrow \mathbb{R}$ a coercive, sequentially weakly lower semicontinuous $C^{1}$ functional, belonging to $\mathcal{W}_{X}$, bounded on each bounded subset of $X$ and whose derivative admits a continuous inverse on $X^{*}$, and $\mathcal{F}: X \rightarrow \mathbb{R}$ a $C^{1}$ functional with compact derivative. Assume that $\Psi$ has a strict local minimum $x_{0}$ with $\mathcal{J}\left(x_{0}\right)=\mathcal{F}\left(x_{0}\right)=0$. Finally, setting

$$
\begin{gathered}
\alpha=\max \left\{0, \limsup _{\|x\| \rightarrow+\infty} \frac{\mathcal{F}(x)}{\mathcal{J}(x)}, \limsup _{\|x\| \rightarrow x_{0}} \frac{\mathcal{F}(x)}{\mathcal{J}(x)}\right\}, \\
\beta=\sup _{x \in \mathcal{J}^{-1}(] 0,+\infty[)} \frac{\mathcal{F}(x)}{\mathcal{J}(x)},
\end{gathered}
$$

and assume $\alpha<\beta$. Then, for each compact interval $[a, b] \in\left(\frac{1}{\beta}, \frac{1}{\alpha}\right)$ (with the conventions $\frac{1}{0}=+\infty, \frac{1}{+\infty}=0$ ) there exists $\nu>0$ with the following property: for every $\lambda \in[a, b]$ and every $C^{1}$ functional $\mathcal{J}: X \rightarrow \mathbb{R}$ with compact derivative, there exists $\gamma>0$ such that, for each $\mu \in[0, \gamma]$, the equation

$$
\mathcal{J}^{\prime}(x)=\lambda \mathcal{F}^{\prime}(x)+\mu \mathcal{H}^{\prime}(x)
$$

has at least three solutions whose norms are less than $\nu$.

\section{REFERENCES}

[1] N. Abatangelo, A remark on nonlocal Neumann conditions for the fractional Laplacian. Preprint arXiv:1712.00320 (2020).

[2] K.B. Ali, M. Hsini, K. Kefi and N.T. Chung, On a nonlocal fractional $p(.,$.$) -Laplacian problem with competing nonlinearities.$ Complex Anal. Oper. Th. 13 (2019) 1377-1399.

[3] A. Alberico, A. Cianchi, L. Pick and L. Slavíková, Fractional Orlicz-Sobolev embeddings. Preprint arXiv:2001.05565 (2020). 
[4] A. Alberico, A. Cianchi, L. Pick and L. Slavíková, On the limit as $s \rightarrow 0^{+}$of fractional Orlicz-Sobolev spaces. Preprint arXiv:2002.05449 (2020).

[5] E. Azroul, A. Benkirane and M. Srati, Existence of solutions for a nonlocal type problem in fractional Orlicz Sobolev spaces. Adv. Oper. Th. 5 (2020) 1350-1375.

[6] A. Bahrouni and V.D. Radulescu, On a new fractional Sobolev space and applications to nonlocal variational problems with variable exponent. Discrete Contin. Dyn. Syst. Ser. S. 11 (2018) 379-389.

[7] S. Bahrouni, H. Ounaies and L.S. Tavares, Basic results of fractional Orlicz-Sobolev space and applications to non-local problems. Topol. Methods Nonlinear Anal. 55 (2020) 681-695.

[8] S. Bahrouni and H. Ounaies, Embedding theorems in the fractional Orlicz-Sobolev space and applications to non-local problems. Discret Cont. Dyn. Syst. 40 (2020) 2917-2944.

[9] S. Bahrouni, Infinitely many solutions for problems in fractional Orlicz-Sobolev spaces. Rochy Mt. J. Math. 50 (2020) 11511173.

[10] A. Bahrouni, S. Bahrouni and M. Xiang, On a class of nonvariational problems in fractional Orlicz-Sobolev spaces. Nonlinear Anal. 190 (2020) 111595.

[11] L. Caffarelli, J.M. Roquejoffre and O. Savin, Nonlocal minimal surfaces. Comm. Pure Appl. Math. 63 (2009) $1111-1144$.

[12] F. Cammaroto and L. Vilasi, Multiple solutions for a nonhomogeneous Dirichlet problem in Orlicz-Sobolev spaces. Appl. Math. Comput. 218 (2012) 11518-11527.

[13] E. Correa and A. de Pablo, Remarks on a nonlinear nonlocal operator in Orlicz spaces. Adv. Nonlinear Anal. 9 (2020) $305-326$.

[14] L.M. Del Pezzo, J.D. Rossi and A.M. Salort, Fractional eigenvalue problems that approximate Steklov eigenvalues. Proc. R. Soc. Edinb. Sect. A 148 (2018) 499-516.

[15] L. Del Pezzo and A.M. Salort, The first non-zero Neumann p-fractional eigenvalue. Nonlinear Anal. Theory Methods Appl. 118 (2015) 130-143.

[16] P. De Nápoli, J. Fernández Bonder and A. Salort, A Pólya-Szegö principle for general fractional Orlicz-Sobolev spaces, To appear in: Complex Var. Elliptic Equations (2020).

[17] S. Dipierro, X. Ros-Oton and E. Valdinoci, Nonlocal problems with Neumann boundary conditions. Rev. Mat. Iberoam 33 (2017) 377-416.

[18] S. El Manouni, H.Hajaiej and P. Winkert, Bounded solutions to nonlinear problems in $\mathbb{R}^{n}$ involving the fractional Laplacian depending on parameters. Minimax Theory Appl. 2 (2017) 265-283.

[19] A. El Khalil, On the spectrum of Robin boundary p-Laplacian problem, Moroccan J. Pure Appl. Anal. 5 (2019) $279-293$.

[20] J. Fernández Bonder and A.M. Salort, Fractional order Orlicz-Sobolev space. J. Funct. Anal. 277 (2019) $333-367$.

[21] J. Fernández Bonder, M. Pérez LLanos and A.M. Salort, A Hölder infinity Laplacian obtained as limit of Orlicz fractional Laplacians. Preprint arXiv: 1807.01669 (2018).

[22] N. Fukagai, M. Ito and K. Narukawa, Positive solutions of quasilinear elliptic equations with critical Orlicz-Sobolev nonlinearity on $\mathbb{R}^{\mathrm{d}}$. Funkcialaj Ekvacioj 49 (2006) 235-267.

[23] U. Kaufmann, J. Rossi and R. Vidal, Fractional Sobolev spaces with variable exponents and fractional. Electron. J. Qual. Theory Differ. Equ. 76 (2017) 1-10.

[24] A. Kristály, M. Mihăilescu and V. Rădulescu, Two non-trivial solutions for a non-homogeneous Neumann problem: an OrliczSobolev space setting. Proc. Roy. Soc. Edinb. 139 A (2009) 367-379.

[25] A. Kufner, O. John and S. Fucik, Function Spaces, Vol. 3. Springer Science Business Media (1979).

[26] J. Lamperti, On the isometries of certain function-spaces. Pacific J. Math. 8 (1958) 459-466.

[27] E. Lindgren and P. Lindqvist, Fractional eigenvalues. Cal. Var. Partial Differ. Equ. 49 (2014) 795-826.

[28] X. Mingqi, V.D. Radulescu, B. Zhang, Fractional Kirchhoff problems with critical Trudinger-Moser nonlinearity. Calc. Var. Partial Differ. Equ. 58 (2019) 57.

[29] G. Molica Bisci, V.D. Radulescu, R. Servadei, Variational Methods for Nonlocal Fractional Problems. Cambridge University Press, Cambridge (2016).

[30] D. Mugnai and E.P. Lippi, Neumann fractional p-Laplacian: Eigenvalues and existence results. Nonlinear Anal. 188 (2019) $455-474$.

[31] D. Mugnai, A. Pinamonti and E. Vecchi, Towards a Brezis-Oswald-type result for fractional problems with Robin boundary conditions. Calc. Var. 59 (2020) 43.

[32] T.C. Nguyen, Three solutions for a class of nonlocalproblems in Orlicz-Sobolev spaces. Appl. Math. Comput. 218 (2013) $1257-1269$

[33] M. Rao and Z. Ren, Applications of Orlicz Spaces, Vol. 250. CRC Press (2002).

[34] B. Ricceri, A further three critical points theorem. Nonlinear Anal. 71 (2009) 4151-4157.

[35] A.M. Salort, Eigenvalues and minimizers for a non-standard growth non-local operator. J. Differ. Equ. 268 (2020) 5413-5439.

[36] R. Servadei and E. Valdinoci, On the spectrum of two different fractional operators. Proc. Roy. Soc. Edinb. Sect. A Math. 144 (2014) 831-855.

[37] B. Volzone, Symmetrization for fractional Neumann problems. Nonlinear Anal. 147 (2016) 1-25.

[38] M. Warma, The fractional Neumann and Robin type boundary conditions for the regional fractional p-Laplacian. Nonlinear Differ. Equ. Appl. 23 (2016) 1.

[39] E. Zeidler, Nonlinear Functional Analysis and Its Applications, Vol. II/B. Springer (1985). 